\title{
Measurement of Highly Non-uniform Residual Stress Fields in Thin Plate Using a New Side Cut Destructive Method
}

\author{
H. K. Kim ${ }^{\mathrm{a}}{ }^{*}$, M. J. Pavier ${ }^{\mathrm{b}}$ and A. Shterenlikht ${ }^{\mathrm{C}}$ \\ Mech Eng Dept, University of Bristol, UK

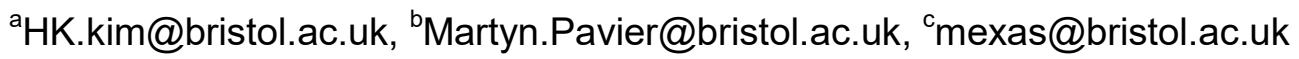

\begin{abstract}
Keywords: Non-uniform Residual Stress, Mechanical Strain Relaxation Technique, Digital Image Correlation, Thin Friction Stir Welding Plate, Plasticity Effect
\end{abstract}

\begin{abstract}
A recently proposed Mechanical Strain Relaxation (MSR) technique for the measurement of residual stress in thin plates is presented. The measurement of residual stress involves making a single straight cut and collecting the relaxation displacement data from the side surface. In this work the method was applied to an Aluminium friction stir welded (FSW) specimen. The cut was introduced with a wire electrical discharge machining. The displacements were recorded with a 3D digital image correlation (DIC) method. The measured FSW residual stress profile agreed well with that measured by Energy Dispersive X-ray Diffraction (EDXRD). It was observed that the amount of plastic strain, caused by stress redistribution during the relaxation process, strongly depends on the direction of the propagation of the cut. In particular, if a cut is propagated along the thickness of a plate, then the effect of plastic flow on the measured residual stress is negligible. Another attractive feature of the method is that it is relatively insensitive to random experimental noise.
\end{abstract}

\section{Introduction}

Over the last two decades a wide range of mechanical strain relaxation (MSR) techniques have been developed and used to measure residual stresses in a wide variety of engineering components. Well known examples of MSR techniques are: hole drilling [1], slitting [2] and contour method [3]. Recently, new technique [4] has been proposed and it follows the general scheme of Mechanical Strain Relaxation (MSR) techniques, i.e. measuring the deformation due to removal of some stressed material in a body and then that measured relaxation is converted to stress.

The main target of the proposed method is that the shape of in-plane residual stress field is highly non-uniform, including discontinuities. Other popular techniques, such as e.g. hole drilling, cannot be used in such cases because they assume stress uniformity within hole diameter [1] and many holes are required to measure residual stress field in relatively large measurement area, leading to complexity of the experiment [5].

During cutting residual stress is redistributed and this might cause plastic deformation where the magnitude of residual stress is close to yield [2]. However, it is shown that this plastic strain on relaxation can be mitigated by choosing the correct propagation of the cut [4]. For this is reason, the technique is attractive in practical application because the measurement can be conducted regardless of the propagation of the cut. It is not always possible for many techniques, for example the slitting method, the propagation of the cut is very important because non-uniform RS can only be measure along depth [2].

In this work, RS field in $3 \mathrm{~mm}$ thick FSW plates have been measured by the proposed method. The application is ideal for the technique because the specimen is very thin and the stress field is non-uniform. The techniques involves a single cut at the midsection of the longitudinal direction of the plate, propagated either from the front to the rear surface, called 'side cut', or from the top to bottom surface, called 'top cut' to observe the difference. 3D DIC were used to measure relaxation displacement on the half width of side surface. Then the residual stress field was reconstructed from the measured relaxation via analytical model.

(c) (1) Content from this work may be used under the terms of the Creative Commons Attribution 3.0 license. Any further distribution of this work must maintain attribution to the author(s) and the title of the work, journal citation and DOI. Published under license by Materials Research Forum LLC. 


\section{Analytical model}

The analytical model for the technique is based on the elastic solution for the problem of selfequilibrated loading at the one end of $2 \mathrm{D}$ semi-infinite strip of width 2c [6], see Fig. 1(a). This analytical model will be employed to reconstruct residual stress field in thin Friction stir welded (FSW) plate from the measured displacement fields on the side surface from 3D DIC, see Fig. 1(b). Other application of the elastic problem to the measurement of residual stress was investigated and described in [4]. Brief overview is given here.

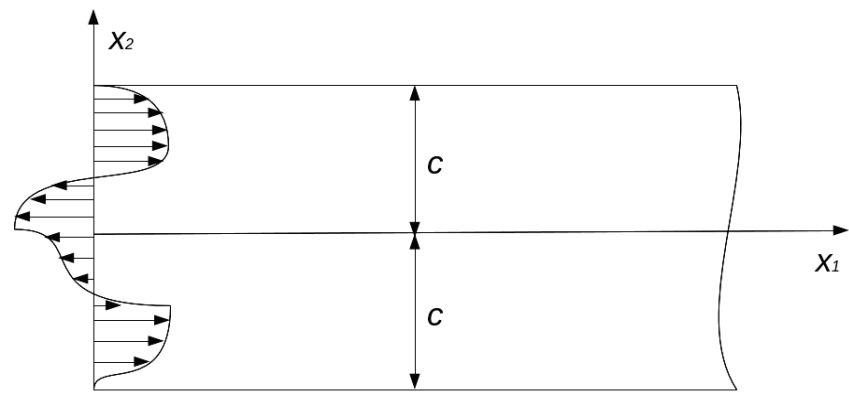

(a)

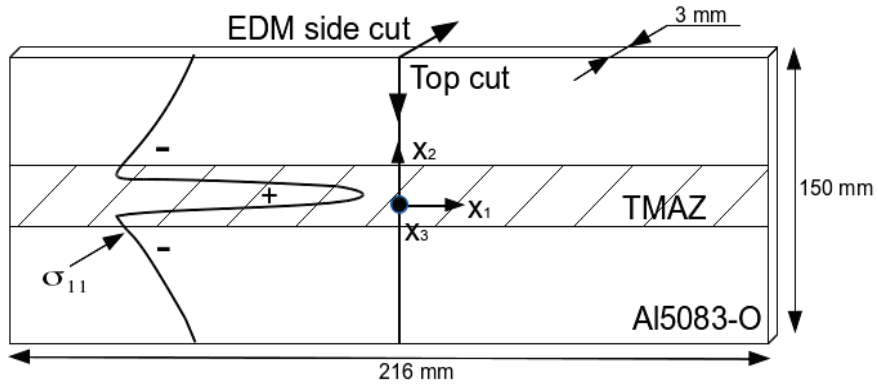

(b)

Fig. 1 : (a) The problem geometry for a $2 D$ semi-infinite rectangular strip, of width $2 c$, with arbitrary self-equilibrated end load [4]. The boundary conditions are: $\pm \boldsymbol{c}: \boldsymbol{\sigma}_{\mathbf{2 2}}=\boldsymbol{\sigma}_{\mathbf{1 2}}=\mathbf{0}$ and $\boldsymbol{x}_{\mathbf{1}}=\mathbf{0}$ : $\int_{-\boldsymbol{c}}^{\boldsymbol{c}} \boldsymbol{\sigma}_{\mathbf{2 2}} \boldsymbol{d} \boldsymbol{x}_{\mathbf{2}}=\int_{-\boldsymbol{c}}^{\boldsymbol{c}} \boldsymbol{\sigma}_{\mathbf{1 2}} \boldsymbol{d} \boldsymbol{x}_{\mathbf{2}}=\mathbf{0}$ and (b) The FSW specimen used in this work. The specimen is cut with wire EDM on the $\boldsymbol{x}_{\mathbf{1}}=0$, the symmetry plane.

The solution uses the two even, f, and odd, g, stress functions which satisfy the bi-harmonic equation:

$$
\mathrm{f}=\mathrm{e}^{-\frac{\gamma x_{1}}{c}}\left(\xi \cos \frac{\gamma x_{2}}{c}+\frac{\gamma x_{2}}{c} \sin \frac{\gamma x_{2}}{c}\right) ; \quad \mathrm{g}=\mathrm{e}^{-\frac{\phi \mathrm{x}_{1}}{c}}\left(\psi \sin \frac{\phi x_{2}}{c}+\frac{\phi x_{2}}{c} \cos \frac{\phi x_{2}}{c}\right)
$$

The boundary conditions, see Fig. 1(a), lead to the following constraints:

$$
\sin 2 \gamma+2 \gamma=0 ; \quad \xi=-\gamma \tan \gamma ; \quad \sin 2 \phi-2 \phi=0 ; \quad \psi=-\phi / \tan \phi
$$

which have infinite number of solutions for dimensionless $\gamma, \xi, \phi, \psi$. A complete stress function, suitable for any arbitrary self-equilibrated stress on the boundary, is constructed as follows:

$$
\theta=\sum_{i=1}^{\infty} a_{i} \operatorname{Re}\left(f_{i}\right)+b_{i} \operatorname{Im}\left(f_{i}\right)+c_{i} \operatorname{Re}\left(g_{i}\right)+d_{i} \operatorname{Im}\left(g_{i}\right)
$$

Using plane stress and small strain assumptions, the surface in-plane displacements can be expressed as follows:

$$
\begin{aligned}
\mathrm{Eu}_{1} & =\sum_{i=1}^{\infty} a_{i} \int \operatorname{Re}\left(\frac{\partial^{2} f_{i}}{\partial x_{2}^{2}}-v \frac{\partial^{2} f_{i}}{\partial x_{1}^{2}}\right) d x_{1}+b_{i} \int \operatorname{Im}\left(\frac{\partial^{2} f_{i}}{\partial x_{2}^{2}}-v \frac{\partial^{2} f_{i}}{\partial x_{1}^{2}}\right) d x_{1} \\
& +\mathrm{c}_{\mathrm{i}} \int \operatorname{Re}\left(\frac{\partial^{2} g_{i}}{\partial x_{2}^{2}}-v \frac{\partial^{2} g_{i}}{\partial x_{1}^{2}}\right) d x_{1}+d_{i} \int \operatorname{Im}\left(\frac{\partial^{2} g_{i}}{\partial x_{2}^{2}}-v \frac{\partial^{2} g_{i}}{\partial x_{1}^{2}}\right) d x_{1}
\end{aligned}
$$




$$
\begin{aligned}
\mathrm{Eu}_{2} & =\sum_{i=1}^{\infty} a_{i} \int \operatorname{Re}\left(\frac{\partial^{2} f_{i}}{\partial x_{1}^{2}}-v \frac{\partial^{2} f_{i}}{\partial x_{2}^{2}}\right) d x_{2}+b_{i} \int \operatorname{Im}\left(\frac{\partial^{2} f_{i}}{\partial x_{1}^{2}}-v \frac{\partial^{2} f_{i}}{\partial x_{2}^{2}}\right) d x_{2} \\
& +\mathrm{c}_{\mathrm{i}} \int \operatorname{Re}\left(\frac{\partial^{2} g_{i}}{\partial x_{1}^{2}}-v \frac{\partial^{2} g_{i}}{\partial x_{2}^{2}}\right) d x_{2}+d_{i} \int \operatorname{Im}\left(\frac{\partial^{2} g_{i}}{\partial x_{1}^{2}}-v \frac{\partial^{2} g_{i}}{\partial x_{2}^{2}}\right) d x_{2}
\end{aligned}
$$

The unknown coefficients in the series expansion of the stress function, $\mathrm{a}_{\mathrm{i}}, b_{i}, c_{i}, d_{i}$, are found from the solution of the standard linear least square (LLS) problem:

$$
\min _{x}|| \boldsymbol{A} \boldsymbol{x}-\boldsymbol{u}||_{2}
$$

where $\mathbf{x}$ is the vector of $4 n$ unknown series coefficients, $\mathbf{u}$ is a vector of $2 m$ displacement values, $\mathbf{A}$ is a $2 m \times 4 n$ matrix of integral functions taken at the locations of the measurement points. To improve LLS stability, the number of measured displacement points, $m$, should be considerably higher than the number of terms in the series expansion, $n$. In practice, hundreds or thousands of data points are needed, which means the method requires a full-field measurement technique.

\section{Experiment}

A $216 \mathrm{~mm} \times 150 \mathrm{~mm} \times 3 \mathrm{~mm}$ plate was manufactured from aluminium alloy $5083-\mathrm{O}, \sigma_{\mathrm{Y}}=$ $145 \mathrm{MPa}$, UTS $=290 \mathrm{MPa}$. The specimen was welded under force control, with a spindle speed of $400 \mathrm{rpm}$ and feed-rate of $200 \mathrm{~mm} / \mathrm{min}$ using a shoulder and pin diameter of 22 and $5 \mathrm{~mm}$, respectively [7]. 3D DIC were used to measure $u_{1}$ and $u_{2}$ relaxation displacements due to the cut.

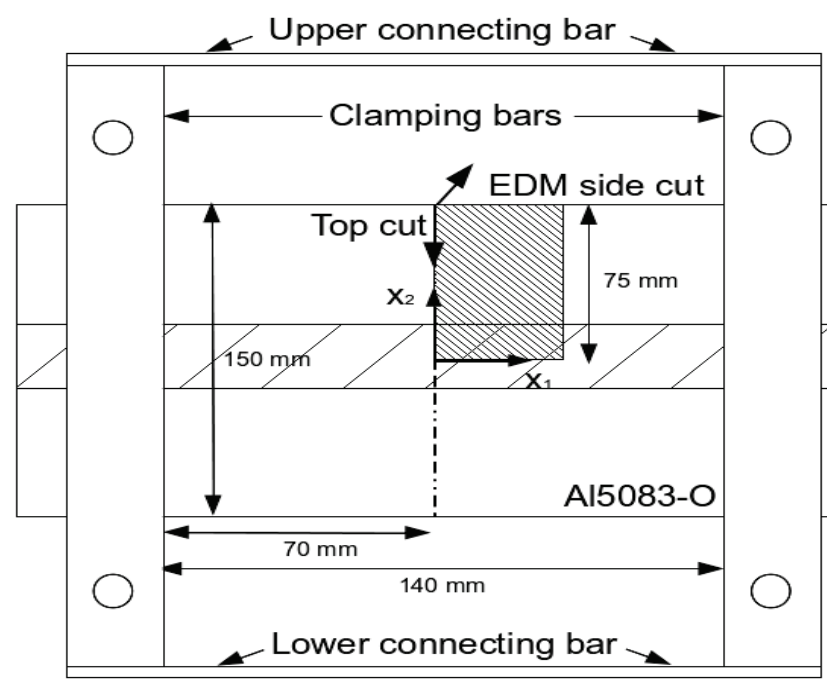

(a)

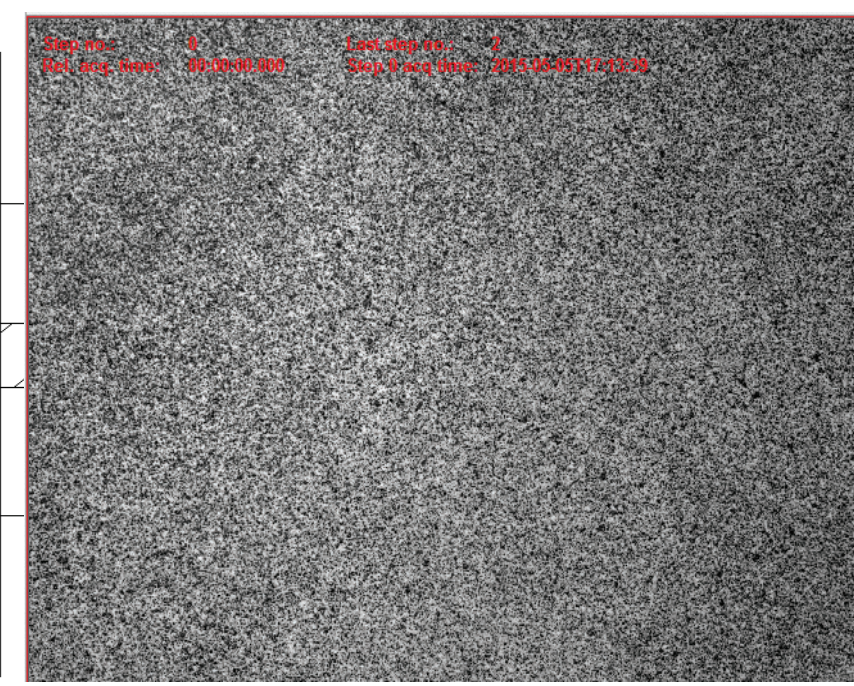

(b)

Fig. 2: Layout of the specimen and the clamps. EDM wire cut cut was done along the dashed line. The fine hatched area indicates the surface fragment where the DIC data was collected. (b) Surface pattern created with matt black and white spray paints.

Clamping was introduced to avoid rigid body movement of the specimen during the cut. The clamping rig was made of four Aluminium bars $\left(\sigma_{Y}=503 \mathrm{MPa}\right.$, UTS $\left.=572 \mathrm{MPa}\right)$ and top and bottom bars were bolted at $70 \mathrm{~mm}$ away from the midsection of the specimen where EDM cutting is carried out, see Fig. 2(a). The clamping bars are connected rigidly to keep the two halves of the cut specimen together after the EDM cut. 
Dantec Dynamics 3D DIC system [8] was used to measure the relaxation. The system consists of two 12-bit $2448 \times 2050$ pixel CCD cameras with zoom lenses and LED light illumination. Taking specimen symmetry into account, only half width of the specimen was recorded to achieve high displacement resolution. The field of view was $76 \times 63 \mathrm{~mm}^{2}$, and the DIC stereo parameters were: the angle of $18.87^{\circ}$ and baseline of $116.34 \mathrm{~mm}$. The size of the pixel was $31 \mu \mathrm{m} /$ pixel. The estimated displacement accuracy was between 0.31 and $0.62 \mu /$ pixel, based on the accuracy of sub-pixel resolution algorithms, typically between 0.02 to 0.01 pixel [9]. 35 and 25 pixel sizes of subset and step size were chosen, giving around $0.78 \mathrm{~mm}$ data point spacing.

To achieve the optimum accuracy of the DIC, suitable surface preparation is critical. The surface should have uniformly distributed suitable size of the random speckle pattern. Both black and white speckle size of at least 3-5 pixels is typically recommended to maintain acceptable noise level [10]. Matt black/white spray paints was found most effective in this work, see Fig 3(b). Additionally, the spray paint pattern can also meet the requirement of the preservation of the surface during the EDM cutting.

Two different propagations of the cut was applied to see the difference in reconstructed residual stress field due to plastic strain produced by different way of stress redistribution: (1) cutting from the front to rear face, called as side cut, and (2) cutting from the top to bottom surface, called as top cut. The EDM wire diameter was $0.25 \mathrm{~mm}$ and the speed of the wire was $18.75 \mathrm{~mm} / \mathrm{min}$ for the top cut and $0.7 \mathrm{~mm} / \mathrm{min}$ for the side cut.

\section{Results}

$\mathrm{u}_{1}$ and $\mathrm{u}_{2}$ surface relaxation displacement fields side cut were obtained from 3D DIC, see Fig. 3. There are few spots where the subsets could not manage to correlate maybe due to surface pattern damage. Unexpected high magnitude of $\mathrm{u}_{2}$ relaxation at the far away from the cut has been observed 3 (a). This might be the error caused by rigid-body rotation during the cut or out of focus in outer region of the lenses.

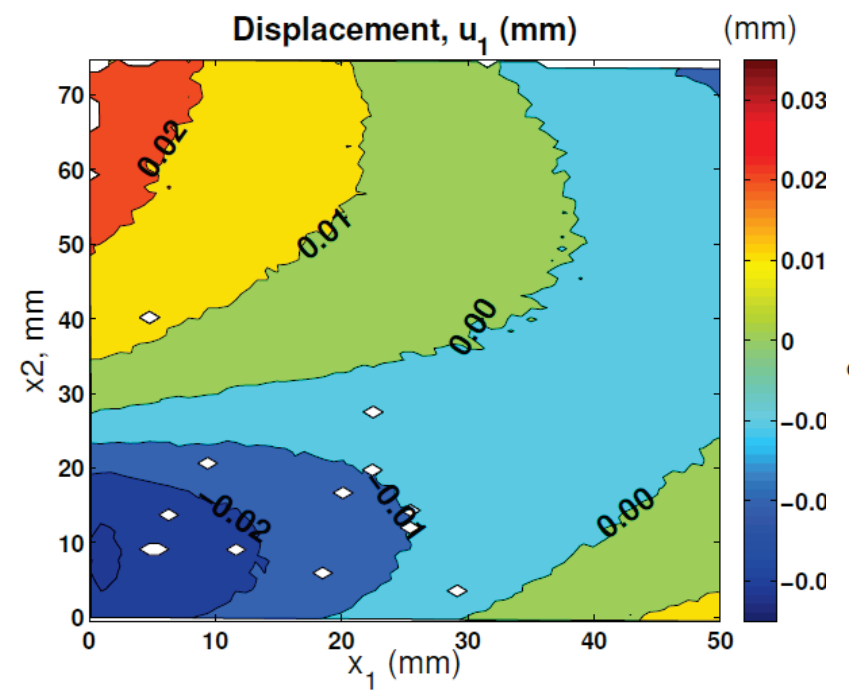

(a)

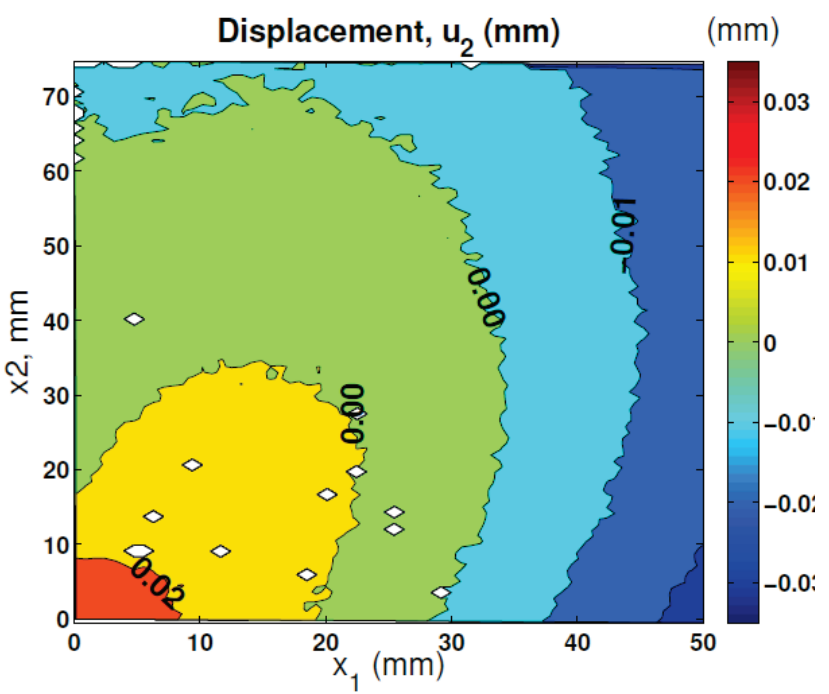

(b)

Fig. 3: $u_{1}(a)$ and $u_{2}$ (b) relaxation displacement results from the front cut. 3D DIC subset and step size are 35 and 25 pixels. The displacement field was collected up to $x_{1}=50 \mathrm{~mm}$ from the cut, $x_{1}=0 \mathrm{~mm}$. 
Fig. 4(a) shows the $u_{1}$ relaxation displacement, obtained from the side and top cut in the region of near the cut. Significant local distortion very close to the cut is seen in the top cut $u_{1}$ field. The magnitude of $u_{1}$ is much lower in the top cut than in the side cut. This might be due to the plastic strain induced during the top cut [4], which leads to large errors in the reconstructed residual stress fields, see Fig. 4(b).

The half width of the residual stress fields reconstructed from measured relaxation displacement fields are compared against EDXRD result, see 4(b). 15 series limits and the displacement data fields from around $\mathrm{x}_{1}=0$ to $5 \mathrm{~mm}$ were used to reconstruct residual stress field to minimise the analytical uncertainty caused by data far away from the cut [4]. Large tail at the tip in the reconstructed residual stress, see Fig. 4(b), has been observed due to the limitation of accurate displacement measurement of DIC at an edge. Also, due to high uncertainty of $\mathrm{u}_{2}$ displacement field from DIC, $\mathrm{u}_{2}$ displacement field was set to zero and it gives more reliable reconstructed residual stress. As a result, although there is oscillation, the reconstructed residual stress field from the front cut agrees very well with EDXRD result, but the result from top cut has large discrepancy with the others due to the distortion near the cut in measured relaxation displacement fields, see Fig. 4(a). It is noted that the EDXRD error bars are taken from [7] and the error bars in the measurement using this method was simulated by adding $5 \%$ random error to the DIC measured displacement field.

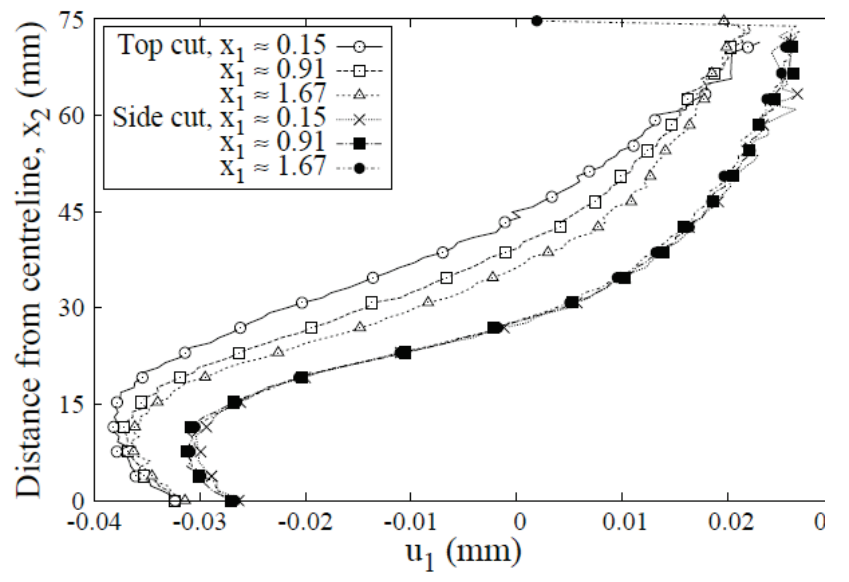

(a)

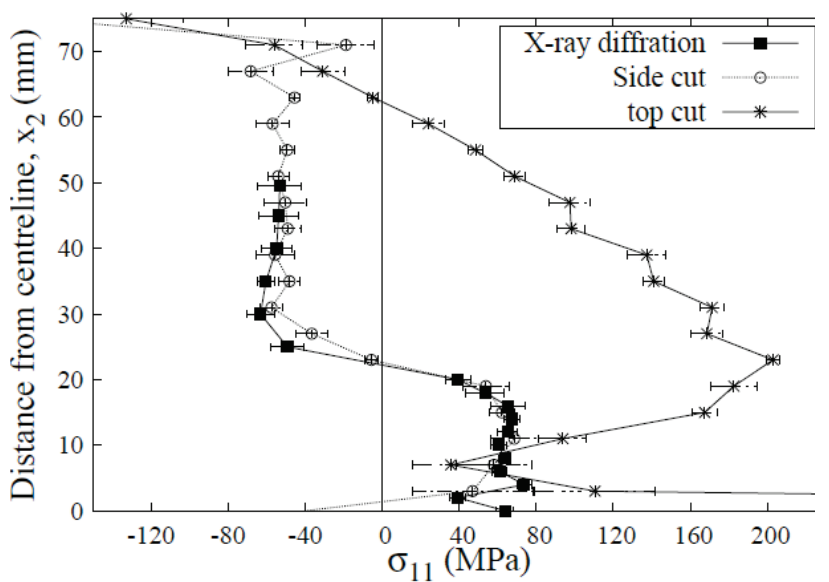

(b)

Fig. 4: (a) $u_{1}$ relaxation displacement profiles near the cutting edge. The magnitude of $u_{1}$ near the cut is lower in the top cut than that in the side cut. (b) Residual stress fields, reconstructed from measured relaxation displacement fields from the side and top cut are compared against EDXRD result [7].

\section{Concluding remarks}

Residual stress profile, induced by FSW in thin A15083-O plate was measured by the new side cut non-uniform residual stress technique. The technique consists of measuring full-field the relaxation displacements due to the cut at midsection of the welding direction of the plate by 3D DIC and reconstructing the residual stress field along the cut from the measured relaxation displacements using analytical model.

Two different cutting propagations were applied to see the plastic flow caused by different behaviour of stress redistribution during the EDM cutting process. In this example, the top cut removed the layer of material with uniform stress, leading to the stress redistribution in the remaining ligament. On the other hand, the side cut removed the layer of material with self-equilibrated stress, 
leading to minimum stress redistribution. As a result, the residual stress field, reconstructed from the side cut had much better agreement with that from EDXRD than that from the top cut.

There are many errors can be added in the relaxation displacement measurement and cause the distortion in the reconstructed residual stress fields. In this example, although the half the width of residual stress field were successfully reconstructed, instability of the reconstruction was shown as oscillation in the residual stress field. The possible causes of instability of the technique could be the limited collection of relaxation displacement data and the rigid body rotation during the cut. Thus, theses errors should be controlled to achieve precise experimental result. Improving 3D DIC measurement system is one of the technical solution. higher resolution of the CCD camera can achieve desire spatial resolution with increased size of the field-of-view.

The clamping is the one of the major issue in the experiment. the relaxation due to the is highly influenced by the clamping, leading to error in reconstruction of residual stress. No clamping is ideal, but this easily cause rigid-body motion. Thus, the optimum clamping strategy must be investigated. the minimum clamping, very far away from the cut, is normally recommended so that the relaxation of the residual stress cannot be affected.

\section{Acknowledgements}

The work was supported by Bristol Alumni Foundation and carried out using the computational facilities of the Advanced Computing Research Centre, University of Bristol. The authors are indebted to Dr. Graeme Horne for providing the specimens and EDXRD data.

\section{References}

[1] G. S. Schajer, Measurement of non-uniform residual stresses using the hole-drilling method, Part I - Stress Calculation Procedures, J. Eng. Mater-T ASME, 110(4):338-343, 1988. http://dx.doi.org/10.1115/1.3226059

[2] W. Cheng and I. Finnie, Residual Stress Measurement and the Slitting Method, Springer, New York, 2007.

[3] M. B. Prime, Cross-sectional mapping of residual stresses by measuring the surface contour after a cut, J. Eng. Mater-T ASME, 123:162-168, 2001. http://dx.doi.org/10.1115/1.1345526

[4] H. K. Kim, H. E. Coules, M. J. Pavier, and A. Shterenlikht. Measurement of highly non-uniform residual stress fields with reduced plastic error, Exp. Mech., 55:1211-1224, 2015. http://dx.doi.org/10.1007/s11340-015-0025-1

[5] W. Xu, J. Liu, and H. Zhu, Analysis of residual stresses in thick aluminum friction stir welded butt joints. Material Design, 32(4):2000-2005, April 2011. http://dx.doi.org/10.1016/j.matdes.2010.11.062

[6] S. Timoshenko and J. N. Goodier, Theory of Elasticity, McGraw-Hill, 3rd edition, 1970.

[7] G Horne, M. J. Peel, and D. J. Smith. Measurement of elastic follow-up for combined applied and residual stresses. In Proceedings of the ASME Pressure Vessels and Piping, PVP2013, 14-18 July 2013. http://dx.doi.org/10.1115/pvp2013-97624

[8] Dantec Dynamics, ISTRA 4D Software Manual Q-400 system, 2012.

[9] B. Pan, H. Xie, B. Xu, and F. Dai. Performance of sub-pixel registration algorithms in digital image correlation, Measurement Science and Technology, 17(6):1615-1621, June 2006. http://dx.doi.org/10.1088/0957-0233/17/6/045

[10] P. Reu. Hidden Components of DIC: Calibration and Shape Function - Part 1. Exp. Techniques, 36(2):3-5, March 2012. http://dx.doi.org/10.1111/j.1747-1567.2012.00821.x 